\title{
9 Spanisch im Beruf: die Tourismusbranche in Berlin
}

Eine der Motivationen zum Erlernen des Spanischen - wenn auch nicht unbedingt die einzige oder die ausschlaggebende - war sowohl bei den Studierenden als auch bei den Lernenden an Volkshochschulen die Verwendbarkeit der Sprache im Beruf. Sprachkenntnisse sind an allererster Stelle im Dienstleistungssektor von Bedeutung, besonders bei einer internationalen und vielsprachigen Kundschaft. In Berlin trifft dies von allen Branchen wahrscheinlich mit am meisten auf die Tourismussparte und das Gastgewerbe zu. Auch dieser Befund ist selbstverständlich nicht für Berlin spezifisch und er gilt genauso für die unterschiedlichsten Regionen und Orte der Welt, die viele Reisende anziehen (vgl. beispielsweise Leslie/Russell 2006).

In diesem Bereich ist der Kontakt zu Muttersprachler^innen des Spanischen und anderer Sprachen äußerst relevant: „Es liegt in der Natur des internationalen Reisens, dass durch das Überschreiten sprachlicher Grenzen Sprecherinnen und Sprecher unterschiedlichster Sprachen miteinander in Kontakt kommen und mehrsprachige Praktiken folglich zur Norm gehören“ (Duchêne/Piller 2011: 135).

Auch im Kontext des Language Making ist das Gastgewerbe bzw. der Tourismus ein interessantes Umfeld. Durch den wiederkehrenden erlebten Kontakt mit Sprecher^innen verschiedenster Sprachen werden Vorstellungen dessen, was eine bestimmte Sprache ausmacht, wie einzelne Sprachen wahrgenommen werden und welchen Stellenwert sie haben permanent neu ausgehandelt und angepasst: „Yet, it is precisely the prevalence of fleeting, symbolic (or metasymbolic) uses of language and other semiotic systems in tourism that makes it such an attractive domain for sociolinguists who are increasingly interested in the fluid and contingent aspects of meaning making." (Heller/Jaworski/Thurlow 2014: 426) Das Spannungsfeld aus der vorübergehenden Begegnung mit den verschiedenen Sprachformen, die kurzzeitig sichtbar und hörbar werden, und der sich damit festigenden Vorstellung von einer Sprache spiegelt exakt wider, wie Language Making funktioniert: Es ist permanent wandelbar und prozesshaft und liefert dennoch in der Wahrnehmung der Betroffenen ein relativ klares, vermeintlich feststehendes Konzept. Der Kontakt mit Spanischsprachigen beispielsweise in Hotels oder Restaurants trägt mit dazu bei, wie das Spanische in Berlin konzeptualisiert wird.

Im Gegensatz zu Spanischsprachigen, die sich längerfristig in Berlin aufhalten und daher in aller Regel mit der Zeit Deutsch lernen, sind kurzzeitige Besucher*innen eine weitaus wichtigere Zielgruppe, bei der die Kommunikation auf Spanisch oder einer gemeinsamen Drittsprache wie Englisch notwendig oder

Ә Open Access. ( 2020 Philipp Krämer, publiziert von De Gruyter. (c) BY Dieses Werk ist lizenziert unter der Creative Commons Attribution 4.0 International Lizenz.

https://doi.org/10.1515/9783110708479-009 
angemessen ist. In den vergangenen Jahren hat die Anzahl von Besucher`innen aus Spanien in Berlin stetig zugenommen, und auch Lateinamerika ist für die Berliner Tourismusbranche ein wichtiger Wachstumsmarkt.

Die Berliner Tourismusstatistik für das Jahr 2016 weist Spanien als Herkunftsland mit der drittgrößten Anzahl von Besucher^innen nach dem Vereinigten Königreich und den USA aus. Danach folgen Italien, die Niederlande und Frankreich. ${ }^{48}$ Nach der Wirtschaftskrise in Spanien stiegen die Besucherzahlen in den letzten Jahren deutlich an, nämlich um 13,9\% zwischen 2015 und 2016, und sogar um 27,7\% im vorausgehenden Jahresabschnitt.

Nimmt man die Herkunftsländer in der Tourismusstatistik zur Grundlage, dann erscheint für eine Gesamtzahl von über 400.000 Gästen im Jahr 2016 das Spanische als ein mögliches und sinnvolles Kommunikationsmittel (Tab. 9.1). Selbstverständlich gilt auch hier, wie im Eingangskapitel in Bezug auf die Nationalitäten der Stadtbewohner^innen, die wichtige Einschränkung, dass aus einem Land nicht zuverlässig auf die Sprachkenntnisse und -verwendung der Personen geschlossen werden kann. Besucher*innen aus Spanien und lateinamerikanischen Ländern können selbstverständlich in vielerlei Hinsicht mehrsprachig sein und müssen nicht notwendigerweise nur Spanisch sprechen; man kann hier an Katalanisch, Baskisch und Galicisch denken, oder an die zahlreichen indigenen Sprachen Amerikas. Man kann aber davon ausgehen, dass für die allermeisten Besucher*innen aus diesen Ländern das Spanische zumindest ein geeignetes Kommunikationsmittel ist, mit dem sie sich gut verständigen können. Im Tourismus erscheint die Verbindung zwischen Nationalität und Sprache etwas zuverlässiger als im Zusammenhang mit Migration, nicht zuletzt weil man eine „tenacious influence of nationality and national identity in tourism discourse" verzeichnen kann (Jaworski/Thurlow 2010: 256). Die Anzahl der Besucher`innen, für die Französisch oder Niederländisch eine günstige Sprachenwahl ist, dürfte sich ungefähr auf demselben Niveau wie die Besucher^innenzahl aus spanischsprachigen Ländern bewegen. ${ }^{49}$

Im Jahr 2016 hatte Berlin insgesamt fünf Millionen ausländische Besucher^innen, wovon 8,3\% auf spanischsprachige Herkunftsländer entfielen. Englisch ist und bleibt selbstverständlich die wichtigste Fremdsprache im Gastgewerbe, nicht

48 Tourismus-Statistik Berlin 2016 (Visit Berlin / Amt für Statistik Berlin-Brandenburg).

49 Für das Französische und das Niederländische ist diese Schätzung noch weitaus schwieriger, weil hier in einigen Fällen die Herkunftsländer durch ihre Mehrsprachigkeitssituation noch weniger Rückschlüsse auf die tatsächlichen Sprachkenntnisse der Menschen zulassen. Dies trifft beispielsweise auf Belgien, die Schweiz oder Kanada zu. 
Tab. 9.1: Anzahl von Besucher^innen (,Ankünfte') aus spanischsprachigen Regionen der Welt im Jahr 2016 (Visit Berlin / Amt für Statistik Berlin-Brandenburg: Tourismusstatistik 2016).

\begin{tabular}{lll}
\hline Spanien & Mittelamerika & Südamerika \\
\hline 310.683 & 30.074 & 76.401 \\
\hline
\end{tabular}

Die Statistik weist Besuche aus Brasilien als eigenständigen Zahlenwert aus, der dementsprechend in der Kategorie Südamerika nicht mitgezählt ist. Damit bleiben fast nur dominant hispanophone Länder übrig, wenn man die Anzahl der Besuche aus Suriname und Guyana als marginal ansieht. Ähnliches gilt für die Kategorie Mittelamerika, die auch die Karibik umfasst. Besuche aus Jamaika, Haiti oder Trinidad und Tobago

u.ä. dürften gegenüber den größeren Ländern wie Mexiko oder Kuba nicht allzu sehr ins Gewicht fallen.

nur durch seine Rolle als Lingua Franca, sondern auch durch die starken Besuchszahlen aus Großbritannien und den Vereinigten Staaten. Ohnehin kann das Englische in Berlin und darüber hinaus längst als eine gängige Arbeitssprache im Berufsleben gelten, jedenfalls für eine breit gefächerte Auswahl an Tätigkeitsbereichen, zu denen der Tourismus zweifellos gehört (Erling/Walton 2007). Daneben ergeben sich für Beschäftigte im Gastgewerbe aber offenbar viele Anwendungsmöglichkeiten für Spanischkenntnisse. Eine Befragung dieser Zielgruppe kann deshalb Aufschlüsse geben über die materielle und instrumentelle Bewertung des Spanischen und anderer präsenter Sprachen.

Darüber hinaus ist der Tourismus ein Bereich, in dem durch interkulturelle Kommunikation und sprachübergreifende Kontakte besonders häufig metasprachliche Auffassungen verhandelt, abgeleitet und gefestigt werden:

Not only does tourism involve face-to-face (or more mediated) forms of visitor-host interaction, like in many other types of service encounters, but the ultimate goods purchased by tourists during their travels are images, lifestyles, memories, and their narrative enactments. [. . .]

[T] ourist - host interactions embody core globalizing processes. It is in communication with each other, in every instant of contact, that hosts and tourists negotiate the nature of their experience, the meanings of culture and place, as well as their own relationships and identities. It is here, too, that many of the meanings of globalization are realized.

(Jaworski/Thurlow 2010: 256, 261)

Die Dienstleister^innen selbst geben dabei selbstverständlich nicht nur Güter an die Besuchenden ab, sondern sie erhalten von diesen auch Feedback in unterschiedlichster Weise - die Begegnung mit anderen Sprachen und Kommunikationsgewohnheiten gehört zu den naheliegenden Erscheinungsformen des Feedbacks. Zugleich kann die Sprache eines großen Kundschaftskreises auch 
auf konkreter materieller Ebene vorteilhaft wirken, wenn damit der Umsatz gesteigert werden kann. Im Tourismus ist die commodification von Sprache(n) deshalb stark spürbar (Jaworski/Thurlow 2010: 258-259). Dieser Hintergrund macht die Beschäftigten im Berliner Gastgewerbe zu einer faszinierenden Zielgruppe, deren Auskünfte zu Spracheinstellungen und Sprachverwendung wertvoll sein können. Wer im Tourismus arbeitet, ist täglich mit den sprachlichen Erscheinungsformen globaler Mobilität konfrontiert und kann Erfahrungen über deren Auswirkungen und Wahrnehmung beitragen.

\subsection{Befragung von Beschäftigten im Berliner Gastgewerbe: Methodisches}

Um näher zu untersuchen, welche Rolle das Spanische im Berliner Gastgewerbe spielt, wurden Ende 2016 Angestellte in Beherbergungsbetrieben und gastronomischen Betrieben nach ihren Sprachkenntnissen, sprachlichen Praktiken und Spracheinstellungen befragt. Im Mittelpunkt stand dabei das Spanische, es wurden aber auch Angaben zu weiteren Sprachen und zur Stellung des Spanischen im Verhältnis zu anderen Sprachen abgefragt. Der Fragebogen war größtenteils quantitativ angelegt, bot aber auch die Gelegenheit zu Freitextkommentaren als Feedback, so dass die Befunde mit qualitativen Daten unterfüttert werden konnten.

Unterstützt wurde die Befragung von der Gewerkschaft Nahrung-GenussGaststätten Berlin-Brandenburg, die einen Fragebogen unter ihren Mitgliedern kommunizierte. Im Unterschied zu den Befragungen unter Spanischlernenden wurden die Beschäftigten im Gastgewerbe durch die Ansprache der Gewerkschaft zur Teilnahme an einem Online-Fragebogen eingeladen. Abgefragt wurde unter anderem die Postleitzahl der Arbeitsstätte. Daten von Beschäftigten außerhalb Berlins oder ohne eindeutig zuzuordnende Lage des Arbeitsplatzes wurden aussortiert, so dass ein Datensatz von 118 Befragten gebildet werden konnte, die in einem Betrieb in Berlin arbeiten.

Einige Fragen des Fragebogens waren identisch mit jenen für die Spanischlernenden oder bieten zielgruppenspezifische Vergleichsmöglichkeiten, der größte Teil unterschied sich jedoch deutlich. Dies liegt in dem zentralen Unterschied begründet, dass man bei den Befragten im Gastgewerbe nicht von vorhandenen Spanischkenntnissen ausgehen konnte. Hier stand vielmehr im Mittelpunkt, die Spracheinstellungen einer Gruppe zu erfassen, die wahrscheinlich häufigen Kontakt zu Spanischsprachigen hat, ohne die Sprache notwendigerweise selbst $\mathrm{zu}$ beherrschen.

Besonderheiten des Fragebogens lagen in den spezifischen Metadaten, die erhoben wurde, neben Alter, Bildungsgrad und Geschlecht etwa die Berufser- 
fahrung im Gastgewerbe, die Art des Betriebs, dessen Zielgruppe und Lage in Berlin oder auch der Aufgabenbereich der Befragten im Betrieb. Erfasst wurden die Kenntnisse verschiedener verbreiteter Sprachen - mit der Möglichkeit, eigene Sprachen hinzuzufügen - und die Häufigkeit der Nutzung anderer Sprachen als des Deutschen im Kontakt mit Gästen. Die Befragten wurden um eine Einschätzung des Nutzens verschiedener Sprachen im Berliner Gastgewerbe gebeten und zudem um Zustimmung bzw. Ablehnung einiger Aussagen zum Umgang mit Mehrsprachigkeit in der Branche. Hierzu zählten Haltungen gegenüber Fremdsprachenkenntnissen und -nutzung im Berufsfeld allgemein sowie Beurteilungen der Bedeutung von Sprachen für die Karriere im Gastgewerbe.

Anschließend wurde der Fragebereich auf das Spanische zugeschnitten. Gefragt wurde nach Kontakten zu spanischsprachigen Gästen und dem Umgang mit ihnen, der Bedeutung des Spanischen in der Berliner Tourismusbranche und allgemeinen Spracheinstellungen zum Spanischen. Der letzte Teil des Fragebogens enthielt zwei Zweige, abhängig von den Spanischkenntnissen der Befragten. Diejenigen, die angaben, kein Spanisch zu sprechen, wurden anhand von Zustimmungsstatements um Einschätzungen gebeten, inwiefern diese Tatsache in ihrem Arbeitsalltag eine Rolle spielt, ob sie vorhaben, Spanisch zu lernen und ob Spanischsprachige Gäste entsprechende Kenntnisse erwarten würden. Der Teil für die Beschäftigten mit Spanischkenntnissen enthielt einen kleine zusätzliche Sektion zum Lernhintergrund (wo, wann und wie das Spanische erworben wurde) sowie weitere Aussagen zur Bewertung im Hinblick auf die Verwendung der Sprache im Berufsalltag und Reaktionen spanischsprachiger Gäste.

Der Wortlaut der einzelnen Items der Befragung ist an den entsprechenden Stellen bzw. bei den Illustrationen in den folgenden Textteilen angegeben. Der vollständige Fragebogen ist im Anhang des Buches wiedergegeben.

\subsection{Ergebnisse der Befragung unter Beschäftigten im Berliner Gastgewerbe}

Das Sample, das sich aus der Beteiligung an der Befragung ergab, stellte sich als gut ausbalancierte Datengrundlage heraus. ${ }^{50}$ Zwar waren männliche Teilnehmer überrepräsentiert (Abb. 9.1), allerdings lassen sich daraus keine zwangsläufigen Verzerrungseffekte ableiten, die in der Interpretation der Daten zu berücksichtigen wären. Befragte im Alter von etwa 20 bis 40 Jahren waren am stärksten ver-

50 Eine erste englischsprachige Auswertung der Daten aus der Befragung im Gastgewerbe findet sich in Krämer (2019b). 


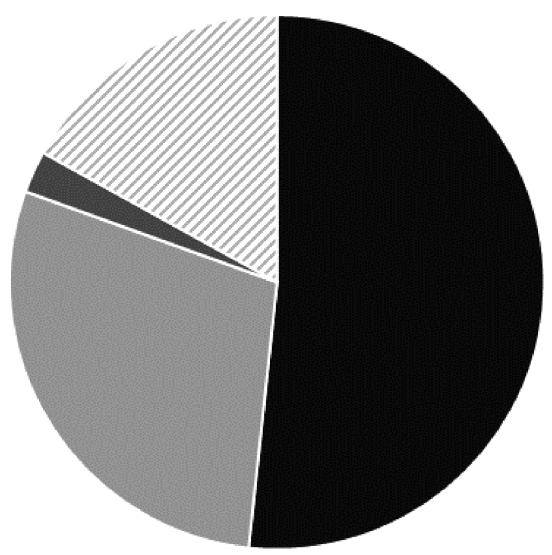

\section{m $\quad$ f andere $/ 2 \mathrm{kA}$}

Abb. 9.1: Geschlechtsverteilung der Befragten im Berliner Gastgewerbe.

treten, was auch ungefähr der Altersstruktur der Beschäftigten entsprechen dürfte: Viele Stellen im Gastgewerbe werden von eher jungen Arbeitnehmer^innen eingenommen, z. B. von Studierenden und anderen nebenberuflich Beschäftigten (Abb. 9.2). Die Befragten hatten eine breit gestreute Berufserfahrung, von Auszubildenden und Anfänger^innen bis hin zu Menschen, die bereits Jahrzehnte in der Branche tätig waren oder schon im Ruhestand sind (Abb. 9.3).

Die Branche, die hier als Gastgewerbe umschrieben ist, umfasst in erster Linie Hotels und andere Beherbergungsbetriebe, sowie Restaurants, Cafés, Bars und andere gastronomische Einrichtungen (Tab. 9.2). Auch im Hinblick auf die unterschiedlichen Betriebstypen erwies sich die Datengrundlage als ausgewogen. Ungefähr gleich viele Befragte arbeiteten im Bereich der Gastronomie und der Beherbergung, jeweils in Betrieben mit unterschiedlicher Ausrichtung. Zahlreiche Befragte gaben mehrere Betriebstypen an, etwa wenn sie in einem Hotel mit eigenem Restaurant arbeiteten oder mehrere Stellen in unterschiedlichen Betrieben kombinierten.

Die befragten Beschäftigten aus dem Berliner Gastgewerbe waren durchweg in den verschiedensten Konstellationen mehrsprachig (Abb. 9.4). Dies verwundert wenig angesichts der Aufgaben und der Kundschaft in der Branche. Ebenso wenig überrascht die Tatsache, dass praktisch alle Befragten muttersprachliche oder fließende Deutschkenntnisse hatten. Wenn auch immer wieder berichtet wird, in gewissen Teilen der Stadt könne mit Angestellten in Cafés oder Restaurants nur noch auf Englisch kommuniziert werden, weil diese kein Deutsch sprächen, sind Deutschkenntnisse offenkundig für den allergrößten 


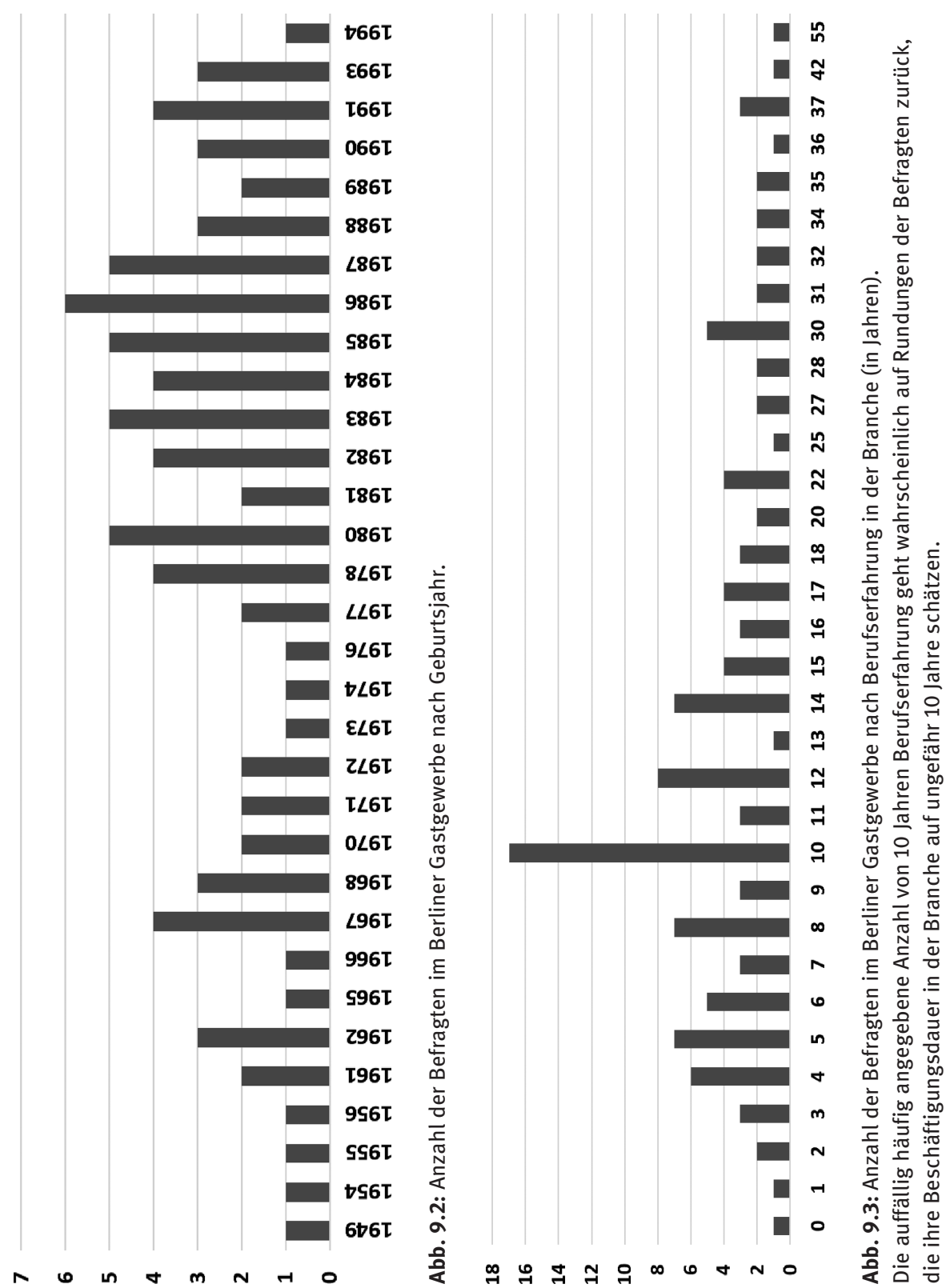


Tab. 9.2: Anzahl der Befragten nach Betriebstypen im Gastgewerbe. (Mehrfachnennungen möglich.)

\begin{tabular}{lcllll}
\hline Beherbergungsbetriebe & \multicolumn{3}{l}{ Gastronomische Betriebe } & \multicolumn{2}{l}{ Andere Betriebe } \\
\hline Hotel 2 Sterne & 7 & Restaurant & 29 & andere & 20 \\
\hline Hotel 3 Sterne & 6 & Bar/Pub/Kneipe & 10 & \\
\hline Hotel 4 Sterne & 26 & Café & 14 & \\
\hline Hotel 5 Sterne & 20 & Imbiss & 5 & \\
\hline Hotel garni & 3 & Club, Disco & 7 & \\
\hline Hostel & 9 & & & & \\
\hline Pension/Gasthof & 0 & & & & \\
\hline Apartmenthotel & 2 & & & & \\
\hline Öffentliches Gästehaus & 1 & & & & \\
\hline B\&B & 0 & & & & \\
\hline Summe & 74 & Summe & & \\
\hline
\end{tabular}

Teil der Beschäftigten noch immer selbstverständlich und unumgänglich. Dasselbe gilt jedoch ebenfalls für das Englische. Muttersprachliche Kenntnisse waren unter den Befragten selten, aber die große Mehrheit gab an, fließend oder auf fortgeschrittenem Niveau Englisch zu sprechen. Praktisch alle anderen konnten sich zumindest verständigen, so dass Gästen das Englische praktisch durchgängig als Kommunikationsmittel zur Verfügung steht.

Bei den weiteren Sprachen bietet sich ein differenziertes Bild. Französisch steht im Hinblick auf seine Verbreitung bei den Befragten klar an dritter Stelle. Deutlich mehr als die Hälfte hatte zumindest Grundkenntnisse des Französischen. Hier scheint die langjährige Fremdsprachenlehre in der Schule mit Französisch als typische zweite Fremdsprache noch abgebildet zu sein. Spanisch dagegen wird relativ ähnlich wie Italienisch und Russisch von einer Minderheit gesprochen. Bei diesen Sprachen gab nur etwa jede ${ }^{\star}$ Dritte an, mindestens Grundkenntnisse zu haben. Die herausgehobene Stellung, die sich das Spanische im Bildungsbereich von der Kita bis zur Erwachsenenbildung bereits erarbeitet hat, wird in der Sprachkompetenz der Angestellten im Gastgewerbe noch nicht sichtbar. Hier reiht sich das Spanische ein in die Kategorie der "dritten“ Fremdsprachen, ohne dass es verbreiteter wäre als das Russische oder das Italienische.

Unerwartet ist, dass nur sehr vereinzelt Sprachen wir Türkisch oder Arabisch angegeben wurden, die im Berliner Gastgewerbe eigentlich sehr häufig anzu- 
treffen sind. Da aber viele Sprecher*innen dieser Sprachen eher in kleineren Familienbetrieben beschäftigt sind, kann man davon ausgehen, dass die meisten nicht von der Einladung zur Teilnahme an der Befragung durch die Gewerkschaft Nahrung - Genuss - Gaststätten erreicht wurden. Es wäre ein lohnenswertes Projekt, die sprachlichen Praktiken und Einstellungen dieser Gruppe noch einmal genauer zu untersuchen und zu vergleichen mit den hier gewonnenen Erkenntnissen.

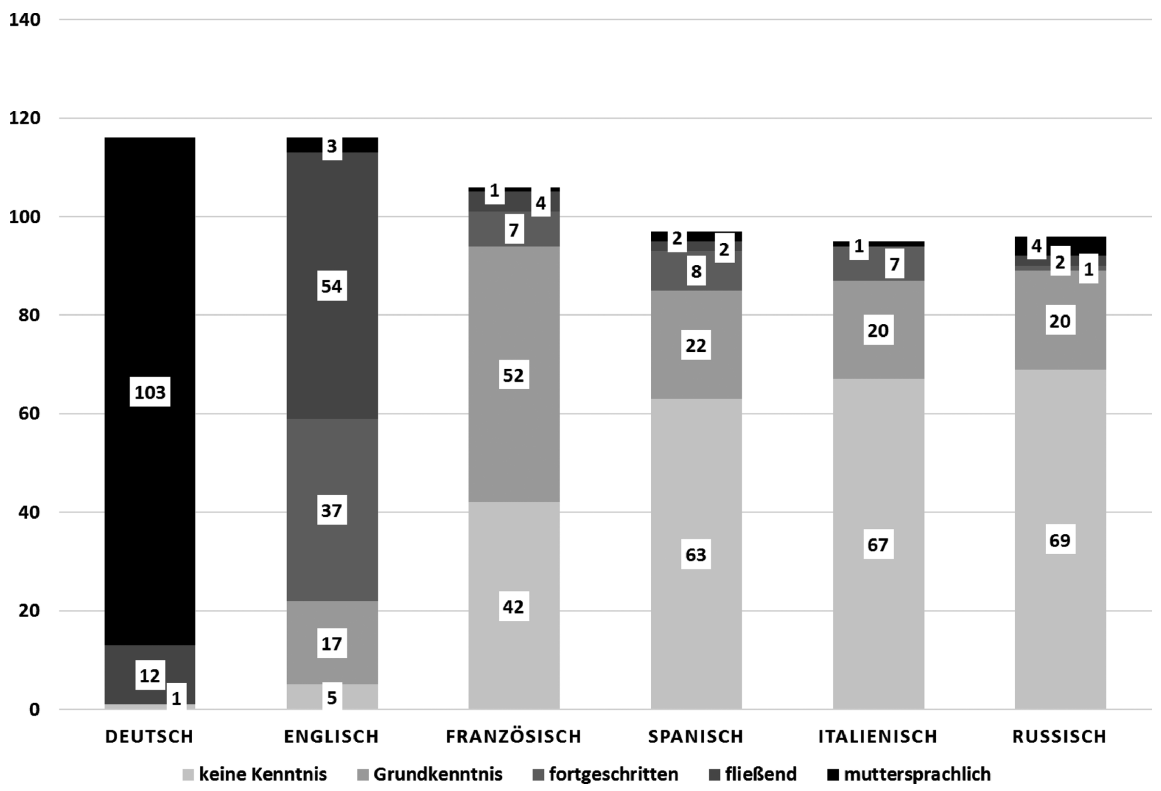

Abb. 9.4: Sprachkenntnisse der Befragten im Berliner Gastgewerbe: Anzahl der Angaben nach Kenntnisstand für die am häufigsten angegebenen Sprachen.

Dass Fremdsprachen im Gastgewerbe absolut notwendig sind, zeigt auch die Tatsache, dass die Befragten nahezu alle im Berufsalltag ihre Sprachkenntnisse anwenden (Abb. 9.5). Die übergroße Mehrheit gab an, praktisch täglich im Kontakt mit Gästen oder Kundschaft eine andere Sprache als Deutsch zu sprechen. Man kann davon ausgehen, dass dies in den allermeisten Fällen Englisch ist, aber auch Kontakt zu spanischsprachigen Gästen kommt regelmäßig vor. Erfahrungen mit der vielsprachigen Kundschaft fasst ein ${ }^{\star}$ Teilnehmer`in so zusammen:

Es ist richtig, dass Berlin viele Touristen aus spanisch sprechenden Länder hat. Allerdings gibt es verwandte Sprachen wie portugiesisch oder italienisch die man inzwischen ebenfalls sehr oft hört. Italienisch zum Beispiel gehört meiner Meinung nach zu den mit am meisten gesprochenen Fremdsprachen in Berlin.

(SiB_337) 


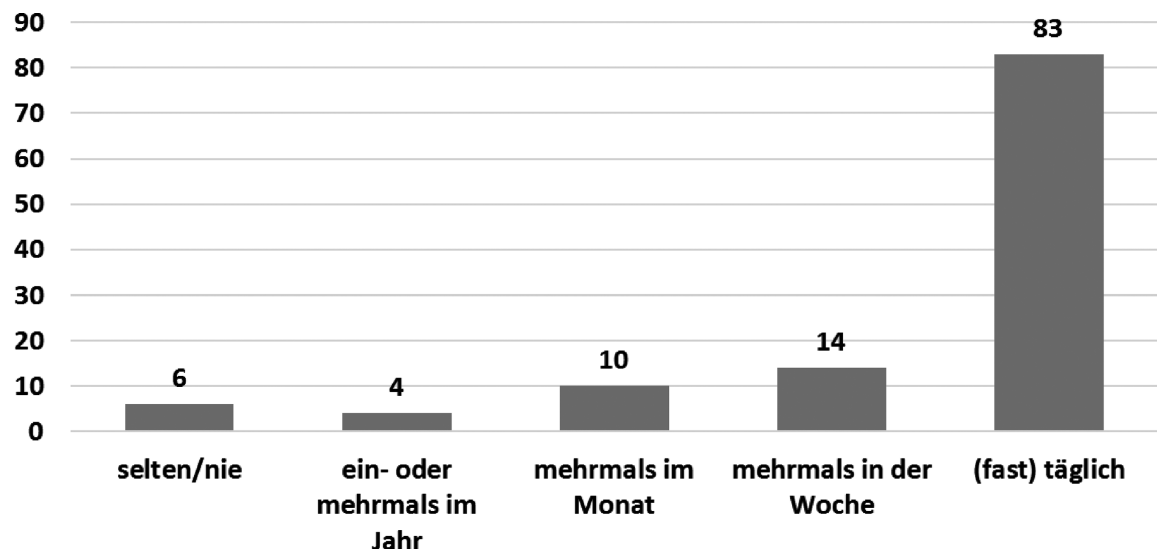

Abb. 9.5: Antworthäufigkeit auf die Frage „Wie oft nutzen Sie mit Gästen eine andere Sprache als Deutsch?“

Kaum jemand unter den Befragten hat nie mit Kundschaft aus spanischsprachigen Ländern zu tun; eine Mehrheit begegnet ihr sogar mehrmals pro Woche oder sogar täglich (Abb. 9.6). An Gelegenheiten, Spanischkenntnisse zu nutzen, mangelt es demnach in diesem Berufsfeld keineswegs. Die Gelegenheiten haben sogar offenbar zugenommen. Die große Mehrheit der Befragten bestätigt, dass der Kontakt mit Gästen aus spanischsprachigen Ländern in den letzten

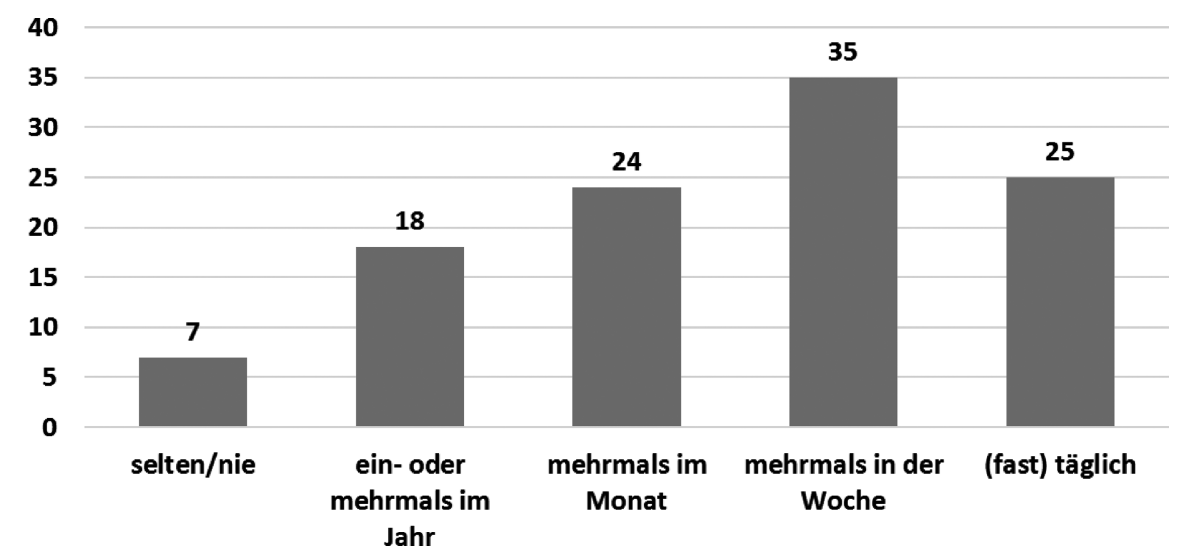

Abb. 9.6: Antworthäufigkeit auf die Frage „Wie oft haben Sie persönlichen Kontakt mit Gästen bzw. Kundschaft aus spanischsprachigen Ländern? (z. B. Spanien, Argentinien, Chile, Venezuela, Kolumbien, Mexiko . . .).“ 
Jahren zugenommen hat (Abb. 9.7). Damit spiegelt sich in den Antworten auch der Trend der Tourismusstatistik wider. Aus ökonomischer Perspektive kann es daher ganz offenkundig attraktiv sein, dieser sehr präsenten Kundschaft auch in ihrer Sprache ein Angebot zu machen. Schließlich kommt den Angaben der Befragten zufolge eine sehr große Anzahl von Betrieben mit diesem Kundenkreis in Berührung, so dass durchaus in gewissem Umfang ein Wettbewerb um Gäste entstehen kann, in dem Dienstleistungen auf Spanisch ein entscheidender Faktor sein könnten.

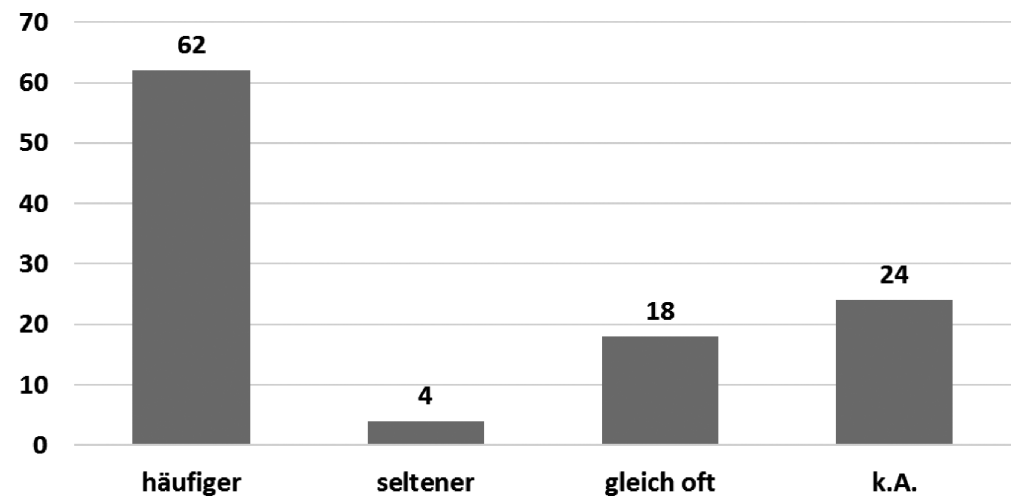

Abb. 9.7: Antworthäufigkeit auf die Frage „Wenn Sie an Ihre Erfahrungen in den letzten 5 bis 10 Jahren zurückdenken, wie hat sich in diesem Zeitraum Ihr Kontakt mit spanischsprachigen Gästen in Berlin entwickelt?“

Der vergleichsweise häufige Kontakt mit spanischsprachigen Gästen könnte möglicherweise diejenigen Beschäftigten, die bisher kein Spanisch sprechen, dazu motivieren, die Sprache zu erlernen. Eine relative Mehrheit der Befragten ließ hierzu aber keine ausgesprochene Neigung erkennen. Zwar gab ein durchaus substanzieller Anteil der Teilnehmer^innen in der Befragung an, Lust zum Spanischlernen zu haben, die meisten lehnten dies aber ab (Abb. 9.8). Hierbei muss auch berücksichtigt werden, dass die Fragestellung darauf abzielte, ob die Befragten „Lust haben, in Zukunft Spanisch zu lernen“. Damit wird also nur eine Neigung abgefragt, nicht aber die feste Absicht. Es ist zu vermuten, dass noch deutlich weniger Befragte angegeben hätten, dass sie es tatsächlich planen, Spanisch zu lernen. Warum dies der Fall ist, obwohl im Berufsalltag für die meisten Beschäftigten offenbar ausreichend Gelegenheit zum Spanischsprechen gegeben wäre, hängt mit den Erfahrungen und Einstellungen der Befragten zusammen, die im nächsten Abschnitt besprochen werden. 
Lust, in Zukunft Spanisch zu lernen.

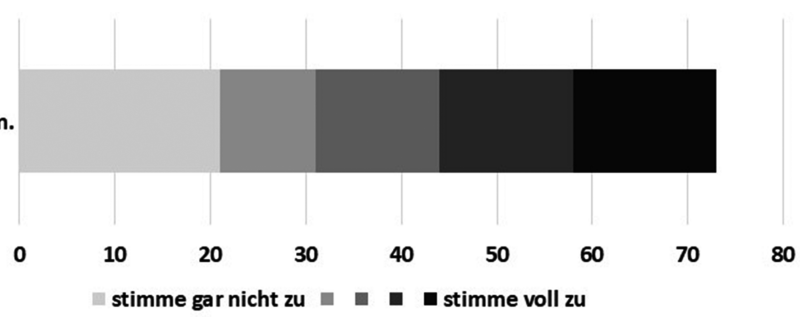

Abb. 9.8: Antworthäufigkeit auf die Aussage „Ich hätte Lust, in Zukunft Spanisch zu lernen.“ (Dieses Item wurde nur denjenigen angezeigt, die vorher angegeben hatten, kein Spanisch zu sprechen).

Wenn Beschäftigte im Gastgewerbe häufig mit nicht-deutschsprachigen Gästen in Kontakt kommen, erscheinen Fremdsprachen grundsätzlich nutzbringend und notwendig für den Berufsalltag:

From the viewpoint of social adaptation, tourist communication represents intercultural communication coining the role of hosts and guests on the one hand, and managing encounters of differences, on the other. In order to accommodate to strangerhood and to mediate between self and the other, it is full of relational strategies [. . .]. (Held 2018: 12)

Schon der Gebrauch einer bestimmten Sprache kann eine solche relational strategy sein. Eine goldene Regel, wann immer möglich die Muttersprache/n der Gäste zu nutzen, mochten viele Befragte allerdings aus der Notwendigkeit zur Anpassung nicht ableiten (Abb. 9.9). Ein großer Anteil der Teilnehmer^innen brachte gegenüber diesem Gedanken eine neutrale Bewertung zum Ausdruck, allerdings sprachen sich insgesamt mehr Befragte dafür als dagegen aus, diesen Grundsatz zu befolgen. Für viele dürfte sich kommunikative Effizienz die Waage halten mit Entgegenkommen gegenüber den Gästen. Es hängt letztendlich sehr stark von den Sprachkenntnissen der einzelnen Beteiligten und auch vom Anliegen der Gäste ab, welche Sprache gewählt wird. In manchen Fällen überwiegt die symbolische Geste, die Sprache der Besucher^innen zu nutzen

Kommunikation in Muttersprache

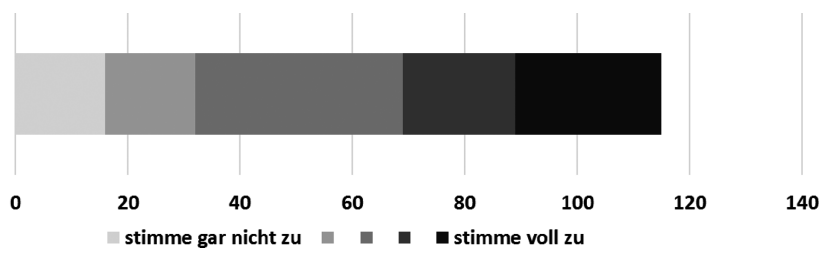

Abb. 9.9: Antworthäufigkeit auf die Aussage „Wer im Gastgewerbe arbeitet, sollte mit Gästen soweit es geht in ihrer Muttersprache kommunizieren.“ 
oder ihnen die Verständigung zu erleichtern. In anderen Fällen kommt es stärker auf Effizienz oder Genauigkeit an, so dass eher eine Sprache gewählt wird, die beide Seiten ausreichend beherrschen. Dies dürfte in den meisten Fällen eher Englisch als Spanisch sein.

Die breite Streuung der Antworten spiegelt letztendlich den Grundsatz wider, dass gerade im Tourismus mit seinen zahlreichen kurzen, häufig zielgerichteten Begegnungen mit interkulturellen Dimensionen sowie klaren Rollen- und Interessensverteilungen sehr viel sprachliche Anpassung erforderlich ist: „Indeed, tourism was expected to be a particular prolific field to show the different ways of how language is effectively subject to permanent adaptation processes as a result of the intentional, mainly economically influenced decisions of choice-making“ (Held 2018: 2).

Verschiedene Sprachen werden als unterschiedlich bedeutsam angesehen. In der Beurteilung der Befragten lagen hier erneut Deutsch und Englisch vorn. Die Unterschiede zwischen beiden Sprachen sind marginal, so dass beide im Prinzip als absolute Notwendigkeit gelten können. Interessanter sind die Einschätzungen der anderen genannten Sprachen. Hier wird Spanisch inzwischen etwas häufiger als wichtig oder sehr wichtig für die Arbeit eingestuft als Französisch (Abb. 9.10). Beide Sprachen werden ungefähr ähnlich eingeschätzt und unterscheiden sich sichtbar von Italienisch oder Russisch.

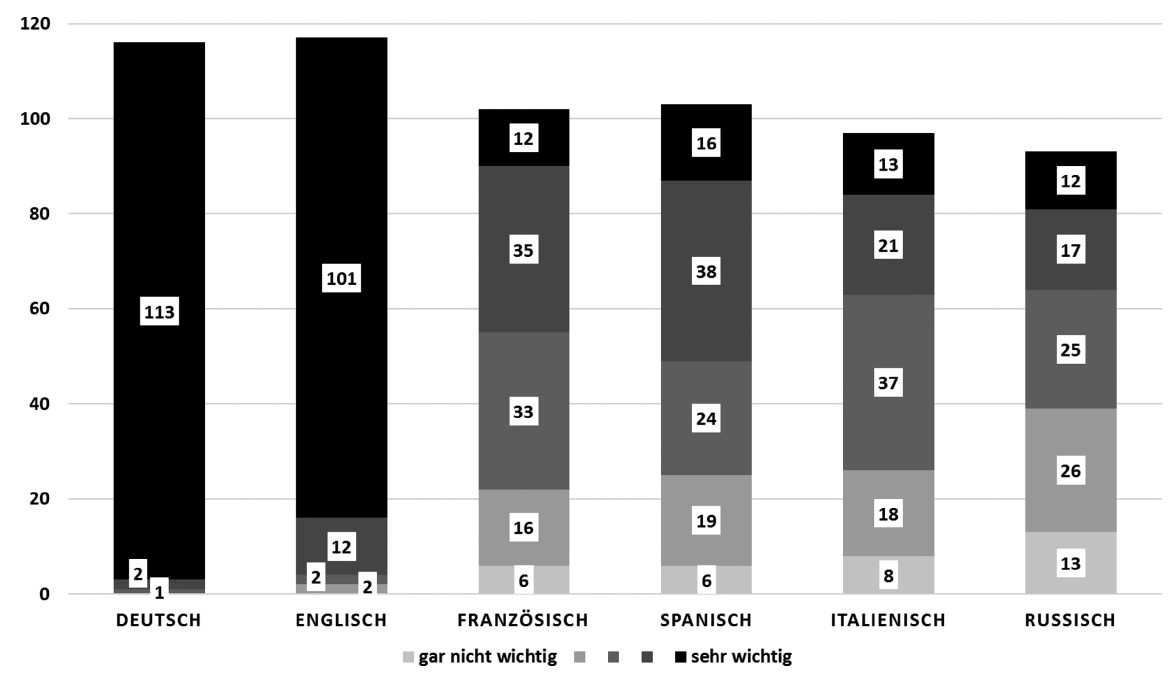

Abb. 9.10: Antworthäufigkeit auf die Frage „Wie wichtig finden Sie Kenntnisse der folgenden Sprachen, um in Berlin im Gastgewerbe arbeiten zu können?“ 
Im Vergleich zu den tatsächlichen Fremdsprachenkenntnissen der Befragten ergibt dies ein etwas ungleichgewichtiges Bild. Offenbar gelten Spanisch und Französisch als ähnlich nützliche Drittsprachen nach Deutsch und Englisch, sie werden aber von den Beschäftigten (noch) nicht gleich oft gesprochen. Die Spanischkenntnisse der Beschäftigten scheinen der gewachsenen Bedeutung der Sprache noch etwas hinterherzuhinken.

In Freitextkommentaren wurden in dem Fragebogen einige weitere Sprachen genannt, die von den Befragten als wichtig oder nützlich eingeschätzt wurden, darunter beispielsweise Chinesisch, Japanisch oder Koreanisch.

Die Werte der Beurteilung von Französisch und Spanisch zeigten eine bedeutende Korrelation, ${ }^{51}$ während diese zwischen Englisch und Französisch bzw. Englisch und Spanisch weitaus schwächer ausgeprägt war. Einige Beschäftigte scheinen demnach generell die Kenntnis von weiteren Sprachen neben Deutsch und Englisch für bedeutsam zu halten, ohne dabei notwendigerweise einen Unterschied zwischen Französisch und Spanisch zu machen. Von der Wichtigkeit des Englischen ist diese Überlegung unabhängig; alle Befragten sind sich einig, dass Englisch nötig ist, sie bewerten lediglich die darüber hinausgehenden Sprachkenntnisse unterschiedlich.

Befragte, die selbst Spanischkenntnisse hatten, schätzten die Sprache nicht notwendigerweise als wichtiger ein als solche, die kein Spanisch sprachen. Zwischen der Beurteilung der Bedeutung des Spanischen und den Sprachkenntnissen bestand keine nennenswerte Korrelation. ${ }^{52}$ Dies ist deshalb interessant, weil man unter Gesichtspunkten des face saving im Rahmen der Befragung an dieser Stelle durchaus einen Zusammenhang hätte vermuten können. Es läge nahe, dass Personen, die eine Sprache gelernt haben, in dieser erworbenen Fähigkeit auch einen Nutzen sehen möchten. Umgekehrt erscheint es vorstellbar, dass man eine Fähigkeit, die man selbst nicht hat, unter Umständen als weniger bedeutsam darstellen möchte. Stattdessen scheinen sich die Befragten unabhängig von ihren Sprachkenntnissen ein Bild von der Bedeutung des Spanischen zu machen und diese Beurteilung aus anderen Faktoren herzuleiten.

Bei der weltweiten Bedeutung des Spanischen zeigten sich die Befragten erstaunlich uneins. Ein ${ }^{\star}$ e Teilnehmer ${ }^{\star}$ in schreibt beispielsweise in ihrem Freitextkommentar am Ende des Fragebogens: „Spanischgrundkenntnisse haben sicher viele Gastronomen, es ist eine wichtige WELTsprache, wichtiger ist aber Englisch.“ (SiB_287) Bei diesem Item des Fragebogens wurden die besonders

51 Kendall's rank correlation: $\mathrm{z}=8.0842, \mathrm{p}=6.259 \mathrm{e}^{-16}$, tau $=0.6767247$.

Pearson's product moment correlation: $\mathrm{t}=11.233, \mathrm{df}=97, \mathrm{p}-\mathrm{value}<2.2 \mathrm{e}^{-16}$, cor $=0.7519247$.

52 Kendall's rank correlation: $\mathrm{z}=2.0916$, $\mathrm{p}$-value $=0.03647$, tau $=0.1853884$. 
polaren Antwortmöglichkeiten an den Rändern der Skala sogar etwas häufiger gewählt als die abgestuften (Abb. 9.11). Offenbar hatten viele der Befragten hier eine dezidierte Meinung: Während einige das Spanische ganz eindeutig als ,Weltsprache‘ einstufen, sehen andere dies überhaupt nicht so.

Spanisch als eine der wichtigsten Sprachen

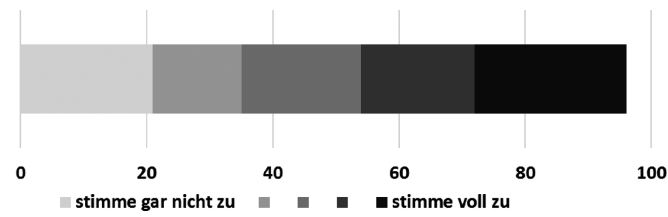

100

Abb. 9.11: Antworthäufigkeit auf die Aussage „Spanisch ist eine der wichtigsten Sprachen der Welt."

Dass man im Gastgewerbe in Berlin eine bessere Dienstleistung bieten kann, wenn man Spanisch kann, bestätigt eine relative Mehrheit der Befragten (Abb. 9.12). Beim Verhältnis zwischen Spanisch und Englisch zeigen sich die Teilnehmer*innen jedoch klar gespalten. Die Antworten zeigen eine fast gleichgewichtige Verteilung bei der Reaktion auf die Aussage, Spanischkenntnisse seien nicht notwendig, wenn man gute Englischkenntnisse hat (Abb. 9.13). Die meisten Befragten beurteilten diesen Gedanken neutral; abgelehnt oder bestätigt wurde die Position von fast exakt gleich vielen Beschäftigten im Gastgewerbe. Erneut zeigen sich die entgegengesetzten Meinungen: Während manche Befragte Englisch als ausreichend ansehen, empfehlen andere es durchaus, zusätzliche Sprachen zu beherrschen.

Bessere Dienstleistung durch Spanisch

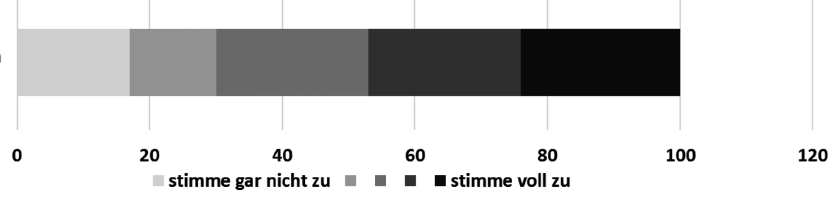

Abb. 9.12: Antworthäufigkeit auf die Aussage „Mit Hilfe von Spanischkenntnissen kann man Gästen eine bessere Dienstleistung bieten.“ 
Englisch statt Spanisch

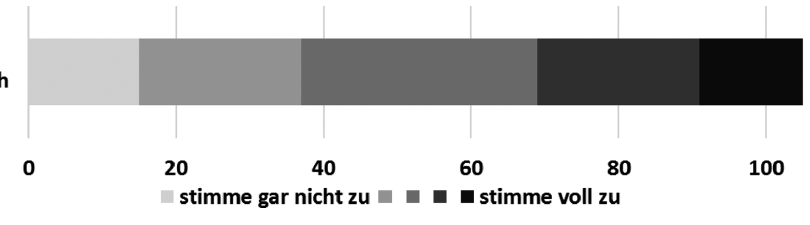

120

Abb. 9.13: Antworthäufigkeit auf die Aussage „Wer im Gastgewerbe gut Englisch spricht, braucht keine Spanischkenntnisse.“

Die beiden unterschiedlichen Ansichten schlagen sich auch in Freitextkommentaren nieder:

Da Berlin viele internationale Touristen besuchen und mittlerweile auch Menschen aus aller Welt hier leben ist es in Berlin in zunehmenden Maße wichtig mehr als Deutsch oder Englisch zu kennen. Allerdings habe ich mich bisher eher von meinen persönlichen Vorlieben zum Sprachen lernen verleiten lassen, nämlich zum Koreanischen. Erfreulicherweise habe ich auch ab und an koreanischstämmige Berliner oder koreanische Touristen die ich in meiner Lieblingssprache bedienen kann.

(SiB_254)

Englisch reicht im Gastgewerbe vollkommen aus.

(SiB_234)

Wer in der Gastronomie in bestimmten Bereichen (Direktion, Verkauf usw.) fließend Englisch sprechen, lesen und schreiben kann, ist schon auf der sicheren Seite!

(SiB_368)

Für den Berufsalltag scheinen die Beschäftigten des Gastgewerbes das Spanische insgesamt als nützlich, aber nicht unbedingt notwendig anzusehen. Bei dieser relativ nuancierten Beurteilung spielen stets auch die unmittelbaren Erfahrungen mit verschiedenen Sprecherinnen und Sprechern eine Rolle: Mit Englisch und Deutsch stehen bereits zwei Sprachen mit großer Reichweite zur Verfügung. Besonders das Englische dürfte von sehr vielen Gästen zumindest einigermaßen beherrscht werden. Spanisch zu sprechen bekommt in diesem Fall den Charakter einer besonderen Dienstleistung, die nicht unbedingt gefordert oder erwartet wird, die aber dafür einen guten Eindruck machen und zur Kundenzufriedenheit oder -bindung beitragen kann. Dementsprechend gab die große Mehrheit der Befragten auch an, dass spanischsprachige Gäste nicht überrascht seien, wenn ihr Gegenüber kein Spanisch spricht (Abb. 9.14). Spanischkenntnisse werden also nicht erwartet, umso stärker kann deshalb der positive Effekt sein, wenn man den Gästen in ihrer bevorzugten Sprache begegnet. Ein*e Teilnehmer^in schreibt hierzu: „Es ist für einen Gast natürlich schön wenn er in seiner Muttersprache angesprochen werden kann. Aber verlangen kann er es nicht“" (SiB_191). Die Sprachverwendung tritt so in einen Zusammenhang mit einer besonderen Performance in der Dienstleistung (Duchêne 2009: 31). Nicht die kommunikativen Bedürfnisse (alleine) sind ausschlaggebend, so dass die Be- 
Ansprache auf Spanisch durch Gäste

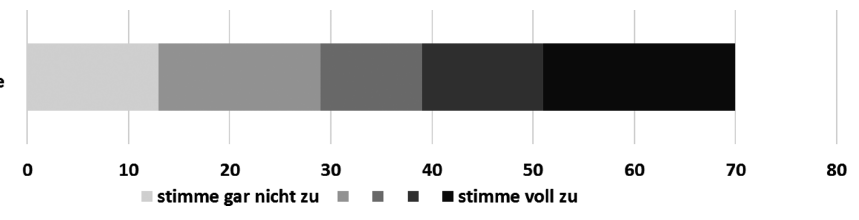

Abb. 9.14: Antworthäufigkeit auf die Aussage „Gäste sprechen mich manchmal auf Spanisch an, obwohl Sie nicht wissen, ob ich Spanisch kann.“

herrschung des Spanischen nicht erwartet werden kann. Stattdessen kann die Verwendung des Spanischen eine vornehmlich pragmatische Funktion erfüllen, indem sie Zugewandtheit, Anpassungsfähigkeit und Dienstleistungsbereitschaft signalisiert. Ein „strategic multilingualism“ im Sinne Duchênes (2009: 38) liegt zumindest was das Spanische betrifft nicht unbedingt auf der Seite der Unternehmen im Sinne einer ganzheitlichen Kommunikationsstrategie für alle Beschäftigten, sondern Mehrsprachigkeit als Strategie wird von einzelnen Beschäftigten situationsbedingt und autonom eingesetzt. Man kann allerdings davon ausgehen, dass diese Strategien durchaus auch im Sinne der Betriebe sind.

Trotz der Befunde zur Erwartungshaltung der Gäste bestätigte fast die Hälfte der Befragten, dass sie häufig auf Spanisch angesprochen würden, auch wenn zuvor noch gar nicht klar war, ob sie Spanisch sprechen (Abb. 9.14). Es scheint daher durchaus eine gewissen Nachfrage nach Dienstleistungen auf Spanisch zu geben und spanischsprachige Gäste würden offenbar das Angebot, Spanisch zu sprechen, tatsächlich des Öfteren annehmen. Dies ist nicht unbedingt eine Selbstverständlichkeit, denn unter Gesichtspunkten der Höflichkeit als Gast oder dem Wunsch nach einer möglichst großen Differenzerfahrung bei einer Auslandsreise mag es bisweilen vorkommen, dass man gerne darauf verzichten möchte, in der Herkunftssprache bedient zu werden. Die bisherige Forschung zeigt jedoch, dass viele Gäste aus Spanien auf Reisen im fremdsprachigen Ausland sehr gerne ihre eigene Sprache nutzen möchten (Goethals 2014; Calvi/Suau-Jiménez 2018: 83-84). Eine Korpusauswertung von Reiseberichten bzw. Reviews von Unterkünften im europäischen Ausland zeigte beispielsweise eine klare Präferenz spanischer Tourist`innen für ihre Muttersprache:

[L]os estudios revelan que la lengua materna juega un papel clave en los relatos que cuentan los turistas hispanohablantes: se refieren más a su lengua materna que los turistas francófonos o germanófonos y sobre todo hacen hincapié en las experiencias positivas, agradeciendo los esfuerzos que realiza la gente local para hablar español. Por otra parte, son bastante negativos sobre sus propios conocimientos lingüísticos, que consideran a menudo deficientes o insuficientes.

(Goethals 2014: 190) 
Für spanischsprachige Kundschaft scheint demnach das Entgegenkommen in der Gastsprache relevanter zu sein als für Kundschaft aus anderen Sprachgemeinschaften.

Neben dieser rein instrumentellen Einschätzung sind an das Spanische auch affektive und materielle Einstellungen geknüpft. Die affektive Beurteilung des Spanischen ist größtenteils unabhängig von der reinen Bedeutung der Sprache im Arbeitskontext und leitet sich für Beschäftigte im Gastgewerbe wie für andere (potenzielle) Sprecher*innen von einer Vielzahl unterschiedlicher Faktoren ab. Dabei spielen sicherlich Begegnungen mit der Sprache im Berufsalltag eine Rolle, z. B. wenn im Umgang mit Gästen häufig Spanisch zu hören ist und man sich in diesen Situationen ein ästhetisches Bild von der Sprache macht. Eindrücke, die in anderen Lebensbereichen entstanden sind, tragen aber mindestens ebenso stark zur affektiven Bewertung einer Sprache bei. Hinzu kommt, dass die Einstellungen einer Person zu einer Sprache sowohl von individuellen Hintergründen und Erfahrungen beeinflusst wird als auch von den sprachideologischen Umgebungsbedingungen, die auf das Individuum einwirken und die Bewertung einer Sprache durch verbreitete Beurteilungsmuster mitformen. Das Verhältnis der einzelnen Faktoren im Anteil an der letztendlich geäußerten Bewertung lässt sich nicht eindeutig quantifizieren, weil sie stets miteinander wechselseitig in Verbindung stehen. Sichtbar ist allerdings sehr wohl, dass eine deutliche Mehrheit der Befragten aus dem Gastgewerbe

Spanisch ist schön

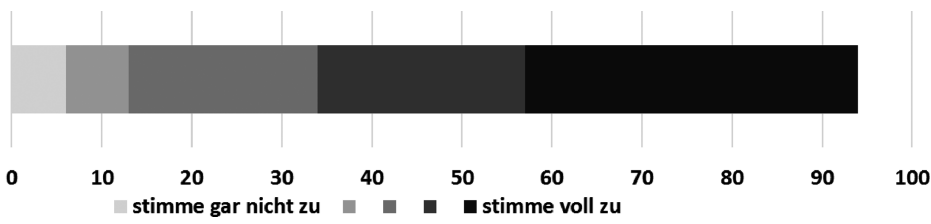

Abb. 9.15: Antworthäufigkeit auf die Aussage „Spanisch ist eine schöne Sprache.“

Spanisch ist schwierig

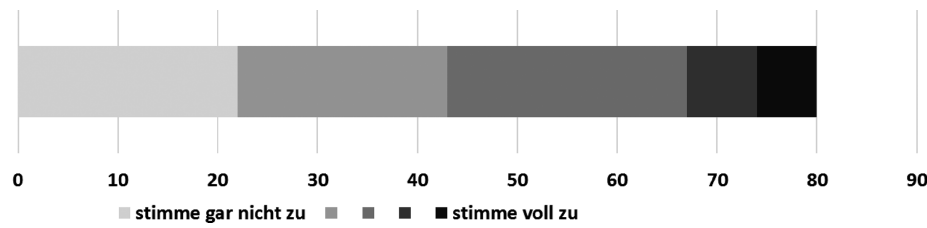

Abb. 9.16: Antworthäufigkeit auf die Aussage „Spanisch ist eine schwierige Sprache.“ 
mit dem Spanischen sehr positive Urteile verbinden (Abb. 9.15 und 9.16). Die Sprache wird von sehr vielen Befragten als schön und auch als nicht besonders schwierig wahrgenommen.

Besonders das zweite Ergebnis kann erstaunen. Spanisch und Französisch sind als romanische Sprachen vom Deutschen strukturell ungefähr gleich weit entfernt; das Französische steht durch seine intensive Kontaktgeschichte zu germanischen Sprachen dem Deutschen im Zweifelsfall sogar noch näher. Französisch gilt vielen Deutschsprachigen als schwer zu erlernende Sprache, besonders im Vergleich zum Englischen. Das Spanische dagegen wird nun von den Befragten als nicht schwierig eingestuft. Zwei Erklärungen bieten sich hierfür an: Viele Befragte gaben an, häufig auch Kontakt mit Gästen aus weiter entfernten Sprachräumen wie z. B. aus Ostasien zu haben. Möglicherweise erschien das Spanische im Vergleich zu dieser Differenzerfahrung daher vertrauter und damit zugänglicher. Eine Rolle spielt zudem sicherlich die insgesamt positive Einstellung, die sich in dem Urteil niederschlägt, Spanisch sei eine schöne Sprache. Eine generelle positive Bewertung der Sprache dürfte ebenfalls dazu beitragen, dass diese als weniger schwierig wahrgenommen wird.

Affektive und instrumentale Einschätzungen einer Sprache sind mehr oder weniger abstrakte Größen. Dem gegenüber steht der materielle Wert, den Kenntnisse einer Sprache mit sich bringen Ein ${ }^{\star} e$ Teilnehmer*in schreibt: „Ich finde englische Sprachkenntnisse in der Gastronomie ausreichend. Wer mehrere Sprachen kann ist natürlich im Vorteil“" (SiB_270). Welcher Art dieser Vorteil ist, bleibt an der Stelle offen. Hier kann entweder eine einfachere Kommunikation oder bessere Einblicke in den kulturellen Hintergrund der Gäste gemeint sein, oder aber Vorteile für die Karriere. Als Qualifikation am Arbeitsmarkt oder als Dienstleistung, die beispielsweise Selbstständige zur Verfügung stellen, kann die Beherrschung einer bestimmten Sprache mehr oder weniger materiellen Ertrag abwerfen. Für das Berliner Gastgewerbe stellt sich deshalb die Frage, ob Spanischkenntnisse einen Effekt auf die Bezahlung der Beschäftigten haben, oder mit anderen Worten: ob die Investition, Spanisch zu lernen, einen Ertrag abwirft.

Die Reaktionen auf diese Frage waren bei den Beschäftigten in der Befragung sehr eindeutig. Nur eine Minderheit bestätigte, dass ihre Vorgesetzten bei der Personalauswahl gezielt auf Fremdsprachenkenntnisse achten (Abb. 9.17). An dieser Stelle wurde noch nicht nach bestimmten Sprachen gefragt, so dass hier auch das Englische mit einbezogen sein kann. Angesichts der Bedeutung von Englischkenntnissen im Gastgewerbe ist dieses Ergebnis zunächst unerwartet, es deckt sich aber mit Erfahrungen beispielsweise aus dem Tourismusgewerbe in der Schweiz: „Individuelle Mehrsprachigkeit wurde als Selbstverständlich betrachtet, als natürliche Bedingung für den Zugang zu diesem Arbeitsmarkt. Die eigentliche sprachliche Herausforderung wurde als eine ,kommunikative“ ver- 
Spanisch bei Einstellung

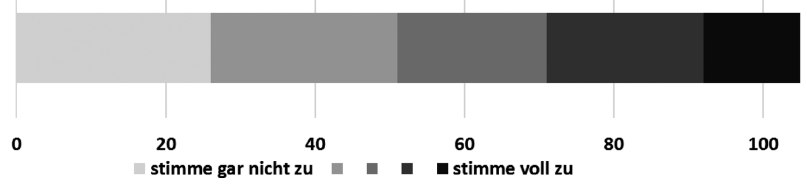

Abb. 9.17: Antworthäufigkeit auf die Aussage „Meine Vorgesetzten achten bei der Einstellung von neuem Personal gezielt auf Fremdsprachenkenntnisse.“

Als alternative Fragestellung konnte ein Item für Beschäftigte mit Personalverantwortung ausgewählt werden. In diesem Fall lautete die vorgegeben Aussage „Als Vorgesetzte/r achte ich bei der Einstellung von neuem Personal gezielt auf Fremdsprachenkenntnisse.“ Dieses Item wurde von deutlich weniger Befragten ausgewählt, die Verteilung war mit derjenigen der parallel gestellten Frage praktisch identisch.

standen“ (Duchêne/Piller 2011: 147). Demnach werden die notwendigen Sprachkenntnisse schlichtweg vorausgesetzt und sie müssen nicht explizit diskutiert werden. Stattdessen steht im Vordergrund die Fähigkeit, mit Gästen bzw. mit der Kundschaft angemessen und kompetent zu kommunizieren, und zwar in den jeweils zur Verfügung stehenden Sprachen: „Gute Kommunikation wird dabei als von jeglicher Sprache unabhängig konzeptionalisiert“ (Duchêne/Piller 2011: 150).

Je nach Betrieb können bei der Bedeutung von Mehrsprachigkeit die unterschiedlichsten Faktoren eine Rolle spielen. In manchen Fällen werden möglicherweise Mitarbeiter^innen ohne oder mit wenig Fremdsprachenkenntnissen eingestellt, wenn genügend weiteres Personal vorhanden ist, das bei Bedarf die Kommunikation übernehmen kann. Diese Vorgehensweise beschreibt auch ein ${ }^{\star}$ Teilnehmer ${ }^{\star}$ in in einem Freitextkommentar:

Wenn jemand nur ,seine“ Sprache sprechen kann gibt es im Hotel jemand der mit ihm ,verhandeln“ kann. Im Laufe der Jahre eignet man sich einige Brocken an, die aber nicht ausreichen um Konversation zu betreiben. Im Übrigen soll man arbeiten und nicht mit den Gästen schwatzen.

(SiB_191)

In anderen Fällen werden vielleicht andere Hilfsmittel verwendet, beispielsweise wenn nur minimale Kommunikation nötig ist. Denkbar sind in der Gastronomie mehrsprachige Speisekarten oder die Nutzung weit verbreiteter Gesten. Ein`e Teilnehmer*in berichtet, dass auch technische Unterstützung wie Übersetzungshilfen verwendet werden:

Die schriftliche Kommunikation mit ausländischen Gästen wird erheblich durch Google Translator vereinfacht. Auch wenn die Übersetzung oft nicht 100\%ig korrekt ist, ist sie jedoch meistens für die Gäste verständlich. Es freut sie sogar, dass man versucht, ihre Sprache zu sprechen.

(SiB_209) 
Eine mögliche Interpretation der Daten ist zudem der Gedanke, dass zumindest Englischkenntnisse als derart selbstverständlich gelten, dass darauf gar nicht mehr gezielt geachtet werden muss. Möglicherweise dachten die Befragten bei dem Begriff „Fremdsprachenkenntnisse“ deshalb eher an andere Sprachen als Englisch, weil dies ohnehin vorausgesetzt wird.

Es erscheint vor diesem Hintergrund logisch, dass die Bedeutung von Spanischkenntnissen für die berufliche Entwicklung im Gastgewerbe als gering eingeschätzt wird, wenn diese bei der Einstellung offenbar wenig Berücksichtigung finden. Auch dies spiegelt die Erfahrungen aus der Schweiz wider: „Zusammenfassend kann gesagt werden, dass mehrsprachige Dienstleistungsinteraktionen Mehrsprachigkeit ironischerweise banalisieren. Die [...] weitverbreitete Annahme, dass Sprachkompetenzen zu Karriereerfolg führen, trifft für die von uns untersuchten Arbeitsplätze in der Tourismusbranche nicht zu“ (Duchêne/Piller 2011: 151). Die Mehrheit der Befragten in Berlin ist daher der Meinung, dass Spanischkenntnisse nicht die Karrierechancen erhöhen (Abb. 9.18).

Verbesserung der Karrierechancen

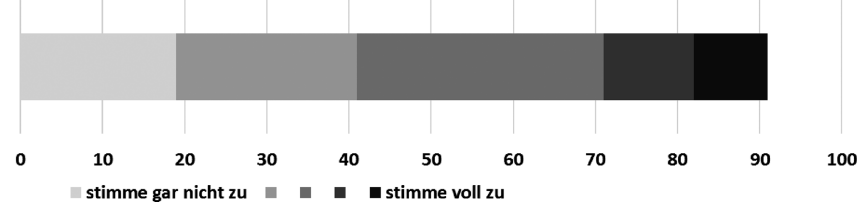

Abb. 9.18: Antworthäufigkeit auf die Aussage „Wenn ich eine neue Stelle suchen müsste, würden Spanischkenntnisse meine Chancen erhöhen.“

Die Nachfrage nach Spanisch am Arbeitsmarkt, zumindest im Gastgewerbe, wird offenbar von den Befragten als gering eingeschätzt. Dies scheint der Beurteilung zu widersprechen, dass Spanischkenntnisse nützlich, aber nicht notwendig sind. In dieser Konstellation könnte das Spanische schließlich einer der Faktoren sein, der bei der Auswahl von Personal den Ausschlag gibt. Während Englisch und Deutsch als unverzichtbar gelten, könnte ein*e Bewerberin mit Spanischkenntnissen die entscheidende Zusatzqualifikation einbringen, die im Arbeitskontext hilfreich ist. Dies scheint aber in der Praxis nicht zuzutreffen. Offenbar sind andere Qualifikationen, die nicht das sprachliche Repertoire der Beschäftigten betreffen, für die Personalgewinnung letztendlich wichtiger.

Dies schlägt sich auch darin nieder, dass Spanischkenntnisse nicht in der Bezahlung der Beschäftigten honoriert werden. Die große Mehrheit der Befragten gab an, dass die Beherrschung des Spanischen keine wirksame Grundlage sei, um ein Gehaltsverbesserung zu fordern (Abb. 9.19). 
Höheres Gehalt

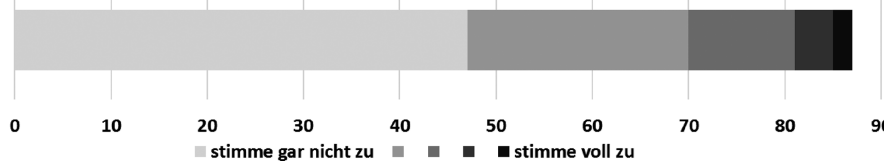

100

Abb. 9.19: Antworthäufigkeit auf die Aussage „Spanischkenntnisse sind im Gastgewerbe ein gutes Argument, um ein höheres Gehalt einzufordern.“

In diesem Zusammenhang muss selbstverständlich berücksichtigt werden, dass das Gastgewerbe ohnehin ein Sektor mit einem niedrigen Lohnniveau ist. Viele Arbeitskräfte in der Branche haben Teilzeitstellen, arbeiten in Nebenoder Gelegenheitstätigkeiten, so dass der Lohndruck auf die Arbeitnehmer^innen generell relativ hoch ist. Eine Verbesserung der Bezahlung lässt sich daher kaum auf Basis einer Qualifikation fordern, die für viele entweder als selbstverständlich (Englisch) oder als verzichtbar (Spanisch) gilt.

Es zeichnet sich ab, dass Sprachkenntnisse im Gastgewerbe auf der materiellen Ebene wenig gewürdigt werden, obwohl sie von den Beschäftigten durchaus als nützlich betrachtet werden. Hier schlägt sich ein weiter verbreitetes Bild von sprachlichen Qualifikationen und auch von sprachbezogenen Berufsbildern nieder, die in weiten Teilen der Gesellschaft als ökonomisch wenig wertvoll betrachtet werden.

Eine stärkere Berücksichtigung des Spanischen am Arbeitsmarkt im Gastgewerbe würde vor allem ein höheres Bewusstsein für den symbolischen Wert der Sprache für die Gäste voraussetzen. Offenbar herrscht bislang vor allem eine Bewertung von Sprachen entsprechend der Notwendigkeit, während die Wirkung von Spanisch als besondere Zusatzdienstleistung nicht materiell gewürdigt wird. Diese kann selbstverständlich dennoch ökonomische Wirkung erzielen, im kleinen Rahmen etwa in Form von Trinkgeldern für guten Service, im größeren Rahmen durch Kundenbindung oder Weiterempfehlung.

Auf materieller Ebene haben Spanischkenntnisse momentan für Beschäftigte im Gastgewerbe keinen nennenswerten ökonomischen Zusatzeffekt. Obwohl die Zeitinvestition und häufig auch die Kosten des Erlernens, z. B. für einen Sprachkurs, in aller Regel von den Beschäftigten getragen werden oder würden, bleibt der Nutzen für sie äußerst gering. Profitieren können von den Spanischkenntnissen eher die Unternehmen, wenn die spanischsprachigen Gäste das Entgegenkommen honorieren. Gäste aus Spanien in US-amerikanischen und britischen Hotels nehmen beispielsweise die Dienstleistung an der Rezeption als besonders höflich wahr, wenn die sprachlichen Hürden niedrig gehalten werden; Hernández-López/Fernández-Amaya (2019: 208) sprechen von „linguistic empathy or, in other words, patience and empathy when there were communicative problems 
due to language barriers.“ Die Sprachbarriere kann insbesondere dann gesenkt werden, wenn das Hotelpersonal ausreichend Kenntnisse der Sprache der Gäste hat (vgl. zum „Payoff“ von Sprachkenntnissen und -lernen in der Privatwirtschaft auch Coulmas 1992: 138-140). ${ }^{53}$ Duchêne/Piller (2011: 151) umschreiben die Problematik folgendermaßen:

Sprachkompetenzen in einer Industrie wie der des Tourismus, die dermaßen von Spracharbeit abhängt, werden vielmehr als selbstverständlich betrachtet und von jeglicher finanzieller Anerkennung ausgeschlossen. Die Unternehmen selbst dagegen profitieren offensichtlich von der Mehrsprachigkeit ihrer Mitarbeiter. [...] Deshalb ist die Frage nach den Profitträgern der Mehrsprachigkeit genauso wichtig wie diejenige nach den „Verlierern der Mehrsprachigkeit“, d. h. den mehrsprachigen Angestellten, deren Spracharbeit essentiell für den Tourismussektor ist, die aber weitgehend von salärieller und statusbezogener Anerkennung ausgeschlossen sind.

Die Befragungsdaten bestätigen diesen Befund eindeutig. Die Gäste haben wiederum ebenfalls einen Nutzen, wenn ihnen die Kommunikation erleichtert wird und sie nicht auf eine Fremdsprache zurückgreifen müssen. Der Nutzen mag nicht ökonomisch zu beziffern sein, ist aber zumindest im Rahmen von eingespartem Aufwand und einem möglicherweise besseren Reiseerlebnis fassbar.

Alles in allem ergibt sich für die Beschäftigten im Gastgewerbe dadurch die paradoxe Situation, dass sie selbst von ihrer eigenen Qualifikation am wenigsten profitieren. Obwohl das Spanische in der Branche nachweislich präsent, nützlich und relevant ist, führen Spanischkenntnisse weder zu besseren Berufsaussichten noch zu einem materiellen Mehrwert für die L2-Sprecherinnen, die es erlernt haben. Der materielle und zum Teil auch immaterielle Nutzen kommt zum allergrößten Teil dem Betrieb und den Gästen zugute, nicht aber den Beschäftigten.

53 Bei Selbstständigkeit und ähnlichen Situationen ist diese Trennung logischerweise nicht aufrecht zu erhalten. In solchen Fällen wäre der Ertrag einer Qualifikation des/der Beschäftigten unmittelbar auch der Ertrag des Betriebs. 
